\title{
Parabolic differential-functional inequalities in viscosity sense
}

\author{
by KRZYSZTof Topolski (Gdańsk)
}

\begin{abstract}
We consider viscosity solutions for second order differential-functional equations of parabolic type. Initial value and mixed problems are studied. Comparison theorems for subsolutions, supersolutions and solutions are considered.
\end{abstract}

1. Introduction. Let $\Omega \subseteq \mathbb{R}^{n}$ be any open domain and $T>0, \tau_{0}, r \in$ $\mathbb{R}_{+}=[0, \infty)$ given constants. Define

$$
\begin{gathered}
\Omega_{r}=\left\{x \in \mathbb{R}^{n}: \operatorname{dist}(x, \Omega) \leq r\right\}, \quad \delta_{0} \Omega=\Omega_{r} \backslash \Omega, \quad \Theta=(0, T) \times \Omega, \\
\Theta_{0}=\left[-\tau_{0}, 0\right] \times \Omega_{r}, \quad \delta_{0} \Theta=(0, T) \times \delta_{0} \Omega, \quad \Gamma=\Theta_{0} \cup \delta_{0} \Theta, \quad E=\Gamma \cup \Theta .
\end{gathered}
$$

(Note that if $\Omega=\mathbb{R}^{n}$ then $\Omega_{r}=\mathbb{R}^{n}, \delta_{0} \Theta=\delta_{0} \Omega=\emptyset$ and $\Gamma=\Theta_{0}$.) Let $D=\left[-\tau_{0}, 0\right] \times B(r)$, where $B(r)=\left\{x \in \mathbb{R}^{n}:|x| \leq r\right\}$ and $|\cdot|$ is the Euclidean norm in $\mathbb{R}^{n}$. For every $z: E \rightarrow \mathbb{R}$ and $(t, x) \in \Theta$ we define a function $z_{(t, x)}: D \rightarrow \mathbb{R}$ by $z_{(t, x)}(s, y)=z(t+s, x+y)$ for $(s, y) \in D$.

For every metric space $X$ we denote by $C(X)$ the class of all continuous functions from $X$ into $\mathbb{R}$ and by $\operatorname{BUC}(X)$ the class of all uniformly continuous and bounded functions from $X$ into $\mathbb{R}$. We will write $\|\cdot\|_{X}$ for the supremum norm. Let $\mathcal{M}(n)$ stand for the space of $n \times n$ real symmetric matrices. Recall that $A \geq B$ if for all $\xi \in \mathbb{R}^{n}$ we have $\langle A \xi, \xi\rangle \geq\langle B \xi, \xi\rangle$ where $\langle\cdot, \cdot\rangle$ denotes the standard inner product. For $A \in \mathcal{M}(n)$ we denote by $\|A\|$ the norm of $A$. Let $F: \Theta \times \mathbb{R} \times C(D) \times \mathbb{R}^{n} \times \mathcal{M}(n) \rightarrow \mathbb{R}$ be a continuous function of the variables $(t, x, u, w, p, A)$ and $g \in C(\Gamma)$ be a given function.

We write $C^{1,2}(\Theta)$ (resp. $C^{1,2}(E)$ ) for the set of all functions from $\Theta$ (resp. E) into $\mathbb{R}$ with continuous derivatives $D_{t} u, D_{x} u, D_{x}^{2} u$.

We consider the initial-boundary value problem

$$
\begin{gathered}
D_{t} z+F\left(t, x, z(t, x), z_{(t, x)}, D_{x} z(t, x), D_{x}^{2} z(t, x)\right)=0 \quad \text { in } \Theta, \\
z(t, x)=g(t, x) \quad \text { in } \Gamma .
\end{gathered}
$$

1991 Mathematics Subject Classification: 35D99, 35B30.

Key words and phrases: viscosity solution, differential-functional equation. 
Even though we say "initial-boundary value problem" it is an initial value problem for $\Theta=(0, T) \times \mathbb{R}^{n}$.

Problem (1), (2) contains as a particular case equations with retarded argument and a few kinds of differential-integral equations.

Definition 1. A function $u \in C(E)$ is called F-subparabolic (resp. F-superparabolic) provided for all $\psi \in C^{1,2}(\Theta)$, if $u-\psi$ attains a local maximum (resp. minimum) at $\left(t_{0}, x_{0}\right) \in \Theta$ then

$$
\begin{aligned}
F\left(t_{0}, x_{0}, u\left(t_{0}, x_{0}\right), u_{\left(t_{0}, x_{0}\right)}, D_{x} \psi\left(t_{0}, x_{0}\right), A\right) \\
\geq F\left(t_{0}, x_{0}, u\left(t_{0}, x_{0}\right), u_{\left(t_{0}, x_{0}\right)}, D_{x} \psi\left(t_{0}, x_{0}\right), B\right)
\end{aligned}
$$

whenever $A \leq B$.

A function $u \in C(E)$ is called $F$-parabolic if $u$ is both $F$-subparabolic and $F$-superparabolic.

Definition 2. A function $u \in C(E)$ is a viscosity subsolution (resp. supersolution) of (1), (2) if $u$ is $F$-subparabolic (resp. $F$-superparabolic) and provided for all $\varphi \in C^{1,2}(\Theta)$, if $u-\varphi$ attains a local maximum (resp. minimum) at $\left(t_{0}, x_{0}\right) \in \Theta$ then

(3) $D_{t} \varphi\left(t_{0}, x_{0}\right)+F\left(t_{0}, x_{0}, u\left(t_{0}, x_{0}\right), u_{\left(t_{0}, x_{0}\right)}, D_{x} \varphi\left(t_{0}, x_{0}\right), D_{x}^{2} \varphi\left(t_{0}, x_{0}\right) \leq 0\right.$

(resp. $D_{t} \varphi\left(t_{0}, x_{0}\right)+F\left(t_{0}, x_{0}, u\left(t_{0}, x_{0}\right), u_{\left(t_{0}, x_{0}\right)}, D_{x} \varphi\left(t_{0}, x_{0}\right), D_{x}^{2} \varphi\left(t_{0}, x_{0}\right) \geq 0\right.$ ) and

$$
u(t, x) \leq g(t, x) \quad(\text { resp. } u(t, x) \geq g(t, x)) \quad \text { in } \Gamma
$$

Definition 3. A function $u \in C(E)$ is a viscosity solution of (1), (2) if $\mathrm{u}$ is both a viscosity subsolution and supersolution of (1), (2).

We denote by $\operatorname{SUB}(F, g), \operatorname{SUP}(F, g), \operatorname{SOL}(F, g)$ the sets of all viscosity subsolutions, supersolutions and solutions of problem (1), (2).

The following is immediate:

REMARK 1. If $u \in C(E) \cap C^{1,2}(\Theta)$ then $u \in \operatorname{SOL}(F, g)$ (resp. $u \in$ $\operatorname{SUB}(F, g), \operatorname{SUP}(F, g))$ if and only if $u$ is a classical solution (resp. subsolution, supersolution) of (1), (2).

This notion of solution was first introduced by M. G. Crandall and P. L. Lions in [4] and [6] for first order differential equations. The best general reference for second order equations is [3].

There are two ways of estimating solutions for parabolic inequalities. We can use one-variable or multi-variable comparison functions. The second method is presented in [5]. This work is devoted to the first. The main result for classical solutions were announced by J. Szarski in [7] and for functionaldifferential equations by the same author in $[8,9]$. Sufficient conditions for the existence of classical solutions for functional-differential equations were given by Brzychczy in $[1,2]$. 
2. Viscosity inequalities. A function $\omega$ is said to satisfy condition " $P$ " if $\omega \in C\left([0, T] \times \mathbb{R}_{+}\right)$is nondecreasing, positive and the right-hand maximum solution of the problem

$$
y^{\prime}(t)=\omega(t, y(t)), \quad y(0)=\sigma,
$$

exists in $[0, T]$. We will denote this solution by $\mu(t, \sigma)$.

Write $a^{+}=\max (0, a), a^{-}=\max (0,-a)$ for $a \in \mathbb{R}$. For $G \subseteq \mathbb{R}^{n+1}$ set $G_{t}=\left\{(s, x) \in G:-\tau_{0} \leq s \leq t\right\}$.

Proposition 1. Let $a>0$ and $h, H \in C([0, a])$. Assume that $h$ is a viscosity solution of $h^{\prime} \leq H$ (i.e. $h$ is a viscosity subsolution of $h^{\prime}=H$ ) in $(0, a)$. Then

$$
h(t) \leq h(s)+\int_{s}^{t} H(\tau) d \tau \quad \text { for } 0 \leq s \leq t \leq a .
$$

The proof can be found in [4], p. 12 .

We will need the following

Assumption 1. 1) There exists a function $\omega$ satisfying condition " $P$ " such that for all $(t, x, u, w) \in \Theta \times \mathbb{R} \times C(D)$, if $u \geq 0$ then

$$
F(t, x, u, w, 0,0) \geq-\omega\left(t, \max \left(u,\left\|w^{+}\right\|_{D}\right)\right) .
$$

2) For every $R>0$ and $|u|,\|w\|_{D} \leq R$,

$$
[F(t, x, u, w, 0,0)-F(t, x, u, w, p, A)]^{+} \rightarrow 0 \quad \text { as } p, A \rightarrow 0
$$

uniformly with respect to $(t, x, u, w) \in \Theta \times \mathbb{R} \times C(D)$.

TheOrem 1. Suppose that $F$ satisfies Assumption 1 and $z \in \operatorname{BUC}(E) \cap$ $\operatorname{SUB}(F, g)$. Then

$$
\left\|z^{+}\right\|_{E_{t}} \leq \mu\left(t,\left\|g^{+}\right\|_{\Gamma_{t}}\right) \quad \text { for } t \in[0, T] .
$$

Proof. Put

$$
M(t)=\left\|z^{+}\right\|_{\bar{\Theta}_{t}}, \quad \bar{M}(t)=\left\|z^{+}\right\|_{\bar{E}_{t}}, \quad M_{0}(t)=\left\|z^{+}\right\|_{\bar{\Gamma}_{t}} \quad \text { for } t \in[0, T] .
$$

Since $z$ is uniformly continuous it follows that $M, \bar{M}, M_{0}$ are continuous. (Note that if $\Omega=\mathbb{R}^{n}$ then $M_{0}(t) \equiv M_{0}(0)$.) It is evident that it suffices to show (6) for $t=T$.

If $M(T) \leq M_{0}(T)$ there is nothing to prove. Suppose that $M(T)>$ $M_{0}(T)$. Since $M(0) \leq M_{0}(0)$ there exists $t^{*} \in[0, T)$ such that

$$
\bar{M}\left(t^{*}\right)=M\left(t^{*}\right)=M_{0}\left(t^{*}\right) \quad \text { and } \quad M(t)>M_{0}(t) \quad \text { for } t \in\left(t^{*}, T\right] .
$$

We will show that

$$
M^{\prime}(t) \leq \omega(t, M(t)) \quad \text { in viscosity sense for } t \in\left(t^{*}, T\right)
$$


(i.e. $M$ is a viscosity subsolution of $\left.y^{\prime}=\omega(t, y)\right)$. Let $\eta \in C^{1}\left(\left(t^{*}, T\right)\right)$ and suppose $M-\eta$ attains a local maximum at $t_{0} \in\left(t^{*}, T\right)$. Since $M$ is nondecreasing it is clear that $\eta^{\prime}\left(t_{0}\right) \geq 0$. We claim that

$$
\eta^{\prime}\left(t_{0}\right) \leq \omega\left(t_{0}, M\left(t_{0}\right)\right) .
$$

Indeed, if $\eta^{\prime}\left(t_{0}\right)=0$ then $(10)$ is obvious. Let $\eta^{\prime}\left(t_{0}\right)>0$. It follows from Lemma 1.4 of [4] that we can find a nondecreasing function $\bar{\eta} \in C^{1}\left(\left[t^{*}, T\right]\right)$ such that $\bar{\eta}^{\prime}\left(t_{0}\right)=\eta^{\prime}\left(t_{0}\right)$ and $(M-\bar{\eta})\left(t_{0}\right)>(M-\bar{\eta})(t)$ for $t \neq t_{0}$. To simplify notation we continue to write $\eta$ for $\bar{\eta}$.

Put $I=\left[t^{*}, T\right]$. Define $\Phi: I \times \bar{\Omega} \rightarrow \mathbb{R}$ by

$$
\Phi(t, x)=z(t, x)^{+}-\eta(t) .
$$

Let $\delta>0$ and let $\left(t^{\prime}, x^{\prime}\right) \in I \times \bar{\Omega}$ be such that $\Phi\left(t^{\prime}, x^{\prime}\right)>\sup \Phi-\delta$. Put

$$
\Psi(t, x)=\Phi(t, x)+2 \delta \xi(x) \quad \text { for }(t, x) \in I \times \bar{\Omega}
$$

where $\xi \in C_{0}^{\infty}\left(\mathbb{R}^{n}\right), 0 \leq \xi \leq 1, \xi\left(x^{\prime}\right)=1,|D \xi|,\left\|D^{2} \xi\right\| \leq 1$ and $D \xi, D^{2} \xi$ are the derivatives of $\xi$. Since $\Psi=\Phi$ outside the support of $\xi$ and $\Psi\left(t^{\prime}, x^{\prime}\right)>$ $\sup \Phi+\delta$ there exists $\left(t_{\delta}, x_{\delta}\right) \in I \times \bar{\Omega}$ such that $\Psi\left(t_{\delta}, x_{\delta}\right)=\sup \Psi$. By the compactness of $I$ we can assume, taking a subsequence if necessary, that $t_{\delta} \rightarrow \bar{t}$ as $\delta \rightarrow 0$.

We claim that $\bar{t}=t_{0}$. Indeed, since

$$
z\left(t_{\delta}, x_{\delta}\right)^{+}-\eta\left(t_{\delta}\right)+2 \delta \geq z(s, x)^{+}-\eta(s) \quad \text { for } t^{*} \leq s \leq t \in I
$$

and $\eta(s) \leq \eta(t)$ we obtain, by $(8)$,

$$
M\left(t_{\delta}\right)-\eta\left(t_{\delta}\right)+2 \delta \geq M(t)-\eta(t) \quad \text { for } t \in I .
$$

Note that in view of $(8), M(t)=\sup \left\{z^{+}(s, x):(s, x) \in \Theta_{t} \backslash \Theta_{t^{*}}\right\}$ for $t \in I$. Letting $\delta \rightarrow 0$ in (14) we get

$$
M(\bar{t})-\eta(\bar{t}) \geq M(t)-\eta(t) \quad \text { for } t \in I,
$$

which means by the definition of $t_{0}$ that $\bar{t}=t_{0}$.

It also follows from (13), (14) (for $t=t_{0}$ ) that

$$
\begin{aligned}
M\left(t_{0}\right)-\eta\left(t_{0}\right) & \geq \limsup _{\delta \rightarrow 0} z\left(t_{\delta}, x_{\delta}\right)^{+}-\eta\left(t_{0}\right) \\
& \geq \liminf _{\delta \rightarrow 0} z\left(t_{\delta}, x_{\delta}\right)^{+}-\eta\left(t_{0}\right) \geq M\left(t_{0}\right)-\eta\left(t_{0}\right),
\end{aligned}
$$

which yields

$$
\lim _{\delta \rightarrow 0} z\left(t_{\delta}, x_{\delta}\right)^{+}=M\left(t_{0}\right) .
$$

Observe now that we may assume that $x_{\delta} \in \Omega$. Indeed, if $x_{\delta} \rightarrow x_{0} \in \delta_{0} \Omega$ then $z\left(t_{0}, x_{0}\right)^{+} \leq M_{0}\left(t_{0}\right)$ and by (15) we have $M\left(t_{0}\right) \leq M_{0}\left(t_{0}\right)$, which contradicts (8). Moreover, by (8), (15) we can also assume that $z^{+}\left(t_{\delta}, x_{\delta}\right)=$ $z\left(t_{\delta}, x_{\delta}\right)>0$. Put

$$
\lambda(t, x)=\eta(t)-2 \delta \xi(x) .
$$


Notice that $z-\lambda$ attains a local maximum at $\left(t_{\delta}, x_{\delta}\right) \in\left(t^{*}, T\right) \times \Omega$. Since

$$
\begin{gathered}
D_{t} \lambda\left(t_{\delta}, x_{\delta}\right)=\eta^{\prime}\left(t_{\delta}\right), \quad D_{x} \lambda\left(t_{\delta}, x_{\delta}\right)=-2 \delta D \xi\left(x_{\delta}\right), \\
D_{x}^{2} \lambda\left(t_{\delta}, x_{\delta}\right)=-2 \delta D^{2} \xi\left(x_{\delta}\right)
\end{gathered}
$$

and $z \in \operatorname{SUB}(F, g)$ in $\Theta \backslash \Theta_{t^{*}}$ we obtain

$$
\eta^{\prime}\left(t_{\delta}\right)+F\left(t_{\delta}, x_{\delta}, z\left(t_{\delta}, x_{\delta}\right), z_{\left(t_{\delta}, x_{\delta}\right)},-2 \delta D \xi\left(x_{\delta}\right),-2 \delta D^{2} \xi\left(x_{\delta}\right)\right) \leq 0
$$

and

$$
\begin{aligned}
\eta^{\prime}\left(t_{\delta}\right) & +F\left(t_{\delta}, x_{\delta}, z\left(t_{\delta}, x_{\delta}\right), z_{\left(t_{\delta}, x_{\delta}\right)},-2 \delta D \xi\left(x_{\delta}\right),-2 \delta D^{2} \xi\left(x_{\delta}\right)\right) \\
& -F\left(t_{\delta}, x_{\delta}, z\left(t_{\delta}, x_{\delta}\right), z_{\left(t_{\delta}, x_{\delta}\right)}, 0,0\right)+F\left(t_{\delta}, x_{\delta}, z\left(t_{\delta}, x_{\delta}\right), z_{\left(t_{\delta}, x_{\delta}\right)}, 0,0\right) \leq 0 .
\end{aligned}
$$

It follows from Assumption 1 that

$$
\eta^{\prime}\left(t_{\delta}\right)-\omega\left(t_{\delta}, \max \left(z\left(t_{\delta}, x_{\delta}\right),\left\|z_{\left(t_{\delta}, x_{\delta}\right)}^{+}\right\|_{D}\right)\right)-A_{\delta} \leq 0
$$

where $A_{\delta} \rightarrow 0$ as $\delta \rightarrow 0$. Hence,

$$
\left.\eta^{\prime}\left(t_{\delta}\right)-\omega\left(t_{\delta},\left\|z_{\left(t_{\delta}, x_{\delta}\right)}^{+}\right\|_{D}\right)\right)-A_{\delta} \leq 0 .
$$

Notice that

$$
\lim _{\delta \rightarrow 0}\left\|z_{\left(t_{\delta}, x_{\delta}\right)}^{+}\right\|_{D}=M\left(t_{0}\right) .
$$

This fact follows from (15) and from the inequality

$$
z\left(t_{\delta}, x_{\delta}\right)^{+} \leq\left\|z_{\left(t_{\delta}, x_{\delta}\right)}^{+}\right\|_{D} \leq z\left(t_{\delta}, x_{\delta}\right)^{+}+2 \delta
$$

where the right-hand estimate is a consequence of (13) (for $t=t_{\delta}$ ). Letting $\delta \rightarrow 0$ in (16) we get (10). It now follows from Proposition 1 (if we put $H(t)=\omega(t, M(t)))$ that

$$
M(t) \leq M\left(t^{*}\right)+\int_{t^{*}}^{t} \omega(s, M(s)) d s, \quad t \in\left[t^{*}, T\right],
$$

which in view of (8) implies

$$
M(t) \leq M_{0}(T)+\int_{0}^{t} \omega(s, M(s)) d s \quad \text { for } t \in\left[t^{*}, T\right] .
$$

Since

$$
M(t) \leq M\left(t^{*}\right)=M_{0}\left(t^{*}\right) \leq M_{0}(T) \quad \text { for } t<t^{*}
$$

inequality (18) holds for $t \in[0, T]$. It follows from standard theorems that

$$
M(t) \leq \mu\left(t, M_{0}(T)\right) \quad \text { for } t \in[0, T] .
$$

Putting $t=T$ we complete the proof.

REMARK 2. If we assume that $\left\|g^{+}\right\|_{\Gamma_{t}} \leq \mu\left(t,\left\|g^{+}\right\|_{\Gamma_{0}}\right)$ for $t \in[0, T]$ then

$$
\left\|z^{+}\right\|_{E_{t}} \leq \mu\left(t,\left\|g^{+}\right\|_{\Gamma_{0}}\right) .
$$


Pr o of. It follows from (17) and (8) that

$$
M(t) \leq \mu\left(t^{*}, M_{0}(0)\right)+\int_{t_{*}}^{t} \omega(s, M(s)) d s \quad \text { for } t \in\left[t^{*}, T\right]
$$

and as a result

$$
M(t) \leq \mu\left(t ; t^{*}, \mu\left(t^{*}, M_{0}(0)\right)\right)=\mu\left(t, M_{0}(0)\right) \quad \text { for } t \in\left[t^{*}, T\right]
$$

where $\mu\left(t ; t^{*}, \mu\left(t^{*}, M_{0}(0)\right)\right)$ denotes the right-hand maximum solution of (16) through $\left(t^{*}, \mu\left(t^{*}, M_{0}(0)\right)\right)$.

Assumption 2. 1) There exists a function $\omega$ satisfying condition "P" such that for all $(t, x, u, w) \in \Theta \times \mathbb{R} \times C(D)$, if $u \leq 0$ then

$$
F(t, x, u, w, 0,0) \leq \omega\left(t, \max \left(u,\left\|w^{-}\right\|_{D}\right)\right) .
$$

2) For every $R>0$ and $|u|,\|w\|_{D} \leq R$,

$$
[F(t, x, u, w, 0,0)-F(t, x, u, w, p, A)]^{-} \rightarrow 0 \quad \text { as } p, A \rightarrow 0
$$

uniformly with respect to $(t, x, u, w) \in \Theta \times \mathbb{R} \times C(D)$.

Corollary 1. Suppose that $F$ satisfies Assumption 2 and $z \in \mathrm{BUC}(E) \cap$ $\operatorname{SUP}(F, g)$ then

$$
\left\|z^{-}\right\|_{E_{t}} \leq \mu\left(t,\left\|g^{-}\right\|_{\Gamma_{t}}\right) \quad \text { for } t \in[0, T] .
$$

Moreover, if we assume that $\left\|g^{-}\right\|_{\Gamma_{t}} \leq \mu\left(t,\left\|g^{-}\right\|_{\Gamma_{0}}\right)$ for $t \in[0, T]$ then

$$
\left\|z^{-}\right\|_{E_{t}} \leq \mu\left(t,\left\|g^{-}\right\|_{\Gamma_{0}}\right) \text {. }
$$

Pr o of. Notice that if $z \in \operatorname{SUP}(F, g)$ that $-z \in \operatorname{SUB}(\widetilde{F},-g)$ where

$$
\widetilde{F}(t, x, u, w, p, A)=-F(t, x,-u,-w,-p,-A) .
$$

It is easy to check that $F$ satisfies Assumption 2 if and only if $\widetilde{F}$ satisfies Assumption 1. Therefore Theorem 1 and Remark 2 imply (20) and (21).

Let us now introduce:

Assumption 3. 1) There exists a function $\omega$ satisfying condition " $P$ " such that for all $(t, x, u, w) \in \Theta \times \mathbb{R} \times C(D)$, if $u \geq 0$ then

$$
F(t, x, u, w, 0,0) \geq-\omega\left(t, \max \left(|u|,\|w\|_{D}\right)\right),
$$

and if $u \leq 0$ then

$$
F(t, x, u, w, 0,0) \leq \omega\left(t, \max \left(|u|,\|w\|_{D}\right)\right) .
$$

2) For every $R>0$ and $|u|,\|w\|_{D} \leq R$,

$$
F(t, x, u, w, p, A) \rightarrow F(t, x, u, w, 0,0) \quad \text { as } p, A \rightarrow 0
$$

uniformly with respect to $(t, x, u, w) \in \Theta \times \mathbb{R} \times C(D)$. 
TheOrem 2. Suppose that $F$ satisfies Assumption 3 and $z \in \operatorname{BUC}(E) \cap$ $\operatorname{SOL}(F, g)$. Then

$$
\|z\|_{E_{t}} \leq \mu\left(t,\|g\|_{\Gamma_{t}}\right) \quad \text { for } t \in[0, T] .
$$

Moreover, if $\|g\|_{\Gamma_{t}} \leq \mu\left(t,\|g\|_{\Gamma_{0}}\right)$ for $t \in[0, T]$ then

$$
\|z\|_{E_{t}} \leq \mu\left(t,\|g\|_{\Gamma_{0}}\right) .
$$

Proof. The proof follows by the same method as for Theorem 1 . The only difference is that we put $|z|$ in place of $z^{+}$. Now, we have

$$
\begin{gathered}
M(t)=\|z\|_{\bar{\Theta}_{t}}, \quad \bar{M}(t)=\|z\|_{\bar{E}_{t}}, \quad M_{0}(t)=\|z\|_{\bar{\Gamma}_{t}} \quad \text { for } t \in[0, T], \\
\Phi(t, x)=|z(t, x)|-\eta(t),
\end{gathered}
$$

and since $\left|z\left(t_{\delta}, x_{\delta}\right)\right|>0$ we consider two cases $z\left(t_{\delta}, x_{\delta}\right)>0$ and $z\left(t_{\delta}, x_{\delta}\right)<0$. Both lead in view of Assumption 3 to (23) and (24).

3. Comparison results. Let $F, \bar{F}: \Theta \times \mathbb{R} \times C(D) \times \mathbb{R}^{n} \times \mathcal{M}(n) \rightarrow \mathbb{R}$ and $g, \bar{g}: \Gamma \rightarrow \mathbb{R}$ be continuous functions.

Assumption 4. 1) There exists a function $\omega$ satisfying condition " $P$ " such that for all $(t, x, u, w, p, A),(t, x, v, z, p, A) \in \Theta \times \mathbb{R} \times C(D) \times \mathbb{R}^{n} \times$ $\mathcal{M}(n)$, if $u \geq v$ then

$$
F(t, x, u, w, p, A)-\bar{F}(t, x, v, z, p, A) \geq-\omega\left(t, \max \left(|u-v|,\left\|(w-z)^{+}\right\|_{D}\right)\right) .
$$

2) For every $R>0$ and $|u|,\|w\|_{D} \leq R, F(t, x, u, w, \cdot, \cdot)$ is continuous uniformly with respect to $(t, x, u, w) \in \Theta \times \mathbb{R} \times C(D)$.

Assumption 5. 1) There exists a function $\omega$ satisfying condition " $P$ " such that for all $(t, x, u, w, p, A),(t, x, v, z, p, A) \in \Theta \times \mathbb{R} \times C(D) \times \mathbb{R}^{n} \times \mathcal{M}(n)$, if $u \geq v$ then

$$
F(t, x, u, w, p, A)-\bar{F}(t, x, v, z, p, A) \geq-\omega\left(t, \max \left(|u-v|,\|w-z\|_{D}\right)\right),
$$

and if $u \leq v$ then

$$
F(t, x, u, w, p, A)-\bar{F}(t, x, v, z, p, A) \leq \omega\left(t, \max \left(|u-v|,\|w-z\|_{D}\right)\right) .
$$

2) $F(t, x, u, w, \cdot, \cdot)$ and $\bar{F}(t, x, u, w, \cdot, \cdot)$ are continuous uniformly with respect to $(t, x, u, w) \in \Theta \times \mathbb{R} \times C(D)$ for every $R>0$ and $|u|,\|w\|_{D} \leq R$.

THEOREM 3. Suppose that $F$ and $\bar{F}$ satisfy Assumption 4 and $u \in$ $\operatorname{BUC}(E) \cap \operatorname{SUB}(F, g), v \in C^{1,2}(\bar{\Theta}) \cap \operatorname{BUC}(E) \cap \operatorname{SUP}(\bar{F}, \bar{g})$. Then

$$
\left\|(u-v)^{+}\right\|_{E_{t}} \leq \mu\left(t,\left\|(g-\bar{g})^{+}\right\|_{\Gamma_{t}}\right) \quad \text { for } t \in[0, T] .
$$

Moreover, if $\left\|(g-\bar{g})^{+}\right\|_{\Gamma_{t}} \leq \mu\left(t,\left\|(g-\bar{g})^{+}\right\|_{\Gamma_{0}}\right)$ for $t \in[0, T]$ then

$$
\left\|(u-v)^{+}\right\|_{E_{t}} \leq \mu\left(t,\left\|(g-\bar{g})^{+}\right\|_{\Gamma_{0}}\right) .
$$


P r o of. It is easily seen that $w^{*}=u-v \in \operatorname{SUB}(F[v], g-\bar{g})$ where

$$
\begin{aligned}
& F[v](t, x, z, w, p, A) \\
& =F\left(t, x, z+v(t, x), w+v_{(t, x)}, p+D_{x} v(t, x), A+D_{x}^{2} v(t, x)\right) \\
& \quad-\bar{F}\left(t, x, v(t, x), v_{(t, x)}, D_{x} v(t, x), D_{x}^{2} v(t, x)\right)
\end{aligned}
$$

satisfies Assumption 1. Theorem 1 and Remark 2 imply the desired assertions.

Similar reasoning yields

Theorem 4. Suppose that $F$ and $\bar{F}$ satisfy Assumption 5 and $u \in$ $\operatorname{BUC}(E) \cap \operatorname{SOL}(F, g), v \in C^{1,2}(\bar{\Theta}) \cap \operatorname{BUC}(E) \cap \operatorname{SOL}(\bar{F}, \bar{g})$. Then

$$
\|u-v\|_{E_{t}} \leq \mu\left(t,\|g-\bar{g}\|_{\Gamma_{t}}\right) \quad \text { for } t \in[0, T] .
$$

Moreover, if $\|g-\bar{g}\|_{\Gamma_{t}} \leq \mu\left(t,\|g-\bar{g}\|_{\Gamma_{0}}\right)$ for $t \in[0, T]$ then

$$
\|u-v\|_{E_{t}} \leq \mu\left(t,\|g-\bar{g}\|_{\Gamma_{0}}\right) .
$$

REMARK 3. For first order equations (with $F, \bar{F}$ not depending on $A$ ) some results, which are not consequences of the above, are presented in [10].

REMARK 4 . The above results may by extended to weakly coupled systems of differential-functional equations.

\section{References}

[1] S. Brzychczy, Chaplygin's method for a system of nonlinear parabolic differentialfunctional equations, Differentsial'nye Uravneniya 22 (1986), 705-708 (in Russian).

[2] - Existence of solutions for non-linear systems of differential-functional equations of parabolic type in an arbitrary domain, Ann. Polon. Math. 47 (1987), 309-317.

[3] M. G. Crandall, H. Ishii and P. L. Lions, User's guide to viscosity solutions of second order partial differential equations, Bull. Amer. Math. Soc. 27 (1992), 1-67.

[4] M. G. Crandall and P. L. Lions, Viscosity solutions of Hamilton-Jacobi equations, Trans. Amer. Math. Soc. 277 (1983), 1-42.

[5] V. Lakshmikantham and S. Leela, Differential and Integral Inequalities, Academic Press, New York, 1969.

[6] P. L. Lions, Generalized Solutions of Hamilton-Jacobi Equations, Pitman, London, 1982.

[7] J. Szarski, Differential Inequalities, PWN, Warszawa, 1967.

[8] —, Sur un système non linéaire d'inégalités différentielles paraboliques contenant des fonctionnelles, Colloq. Math. 16 (1967), 141-145.

[9] -, Uniqueness of solutions of mixed problem for parabolic differential-functional equations, Ann. Polon. Math. 28 (1973), 52-65. 
[10] K. Topolski, On the uniqueness of viscosity solutions for first order partial differential-functional equations, ibid. 59 (1994), 65-75.

Institute of Mathematics

University of Gdańsk

Wita Stwosza 57

80-952 Gdańsk, Poland

E-mail: matkt@ksinet.univ.gda.pl

Reçu par la Rédaction le 10.4.1995 